\title{
SYNTHESIS AND ANALYSIS OF RADAR SIGNAL CLASSIFICATION ALGORITHMS
}

\author{
Leonid G. Dorosinskiy \\ Ural Federal University Named After the First \\ President of Russia B.N. Yeltsin \\ Institute of Radioelectronics and \\ Information Technologies \\ 620002, Ekaterinburg, RUSSIAN FEDERATION
}

\begin{abstract}
In the process of solving a wide range of tasks concerning the Earth surface remote sensing and its state monitoring, the main role is played by the algorithm of the surface image forming and the algorithm of images and their fragments classification as well. From the statistical point of view the solution is based on the maximum-likelihood method. The paper presents analytical equations for likelihood coefficients and the structural scheme of their forming in the solution of radar signal recognition. To analyze the efficiency of the proposed algorithms are found boundary equations for probability calculating of correct signals with the usage of Chernoff- Kailath ratios. This ratios evaluate the upper and lower probability boundaries of correct and incorrect decisions in the case of classification of optional class number from different type surfaces.
\end{abstract}

AMS Subject Classification: 11A05, 60G35, 65D15, 93E10

Key Words: recognition, algorithm, probability

\section{Introduction}

Among the tasks of radio astronomy and the remote sensing of the surface, the key role is fulfilled by the optimal or close to the processing of received data with

$\begin{array}{lr}\text { Received: } & \text { August 1, } 2018 \\ \text { Revised: } & \text { August 18, } 2016 \\ \text { Published: } & \text { September 30, } 2016\end{array}$

(c) 2016 Academic Publications, Ltd. url: www.acadpubl.eu 
the target of the efficient problem solution realization of the recognition of the observable signal class. The efficient problem solution of the class recognition [1], [2], [3], [4] may be received within the classical theory of multiple-choice check of the statistical hypotheses. According to one of them the vector of received fluctuations $\bar{U}(t)$ is generated only by noise. The other hypotheses correspond to the observation of different class signals. The general number of possible classes- $M$, the hypotheses number $-(M+1)$.

\section{Results and Discussion}

The traditional task solution of the multiple-choice hypotheses check leads to the device structure of the received signals processing that consists of M parallel channels of likelihood ratio forming or its logarithm

$$
l_{k}[\bar{U}(t)]=\ln \left\{L_{k}[\bar{U}(t)]\right\},
$$

and the resolver which $M$ input receives $l_{k}$ values. The resolver chooses one of $M$ signals. The solution depends on the selected quality criterion. Basing on the maximum likelihood criterion the solution is the hypothesis with the maximum $l_{k}$ value. Any case the most practical interest is in the procedure of likelihood ratio formation and the device structural chart that implements this ratio.

The sufficient statistics for making the solution is the vector composed from the likelihood ratio for the each of $M$ competing hypotheses. The logarithm of the likelihood ratio for $k$-hypothesis is written as follows:

$$
\begin{aligned}
l_{k}[\bar{U}(t)] & =0,5\left\{\iint \bar{U}^{*}(t) Q_{0}(t, u) \bar{U}(u) d t d u-\right. \\
& \left.-\iint\left[\bar{U}^{*}(t)-\bar{U}_{k \sigma}^{*}(t)\right] Q_{k}(t, u)\left[\bar{U}(u)-\bar{U}_{k \sigma}(u)\right] d t d u-\ln \left(\frac{K_{0}}{K_{k}}\right)\right\}
\end{aligned}
$$

where $K_{0}$ and $K_{k}$ - the normalizing factors of the functional density of probability distribution for the case just the noise observation and $k$-signal against the noise background; $Q_{0}(t, u), Q_{k}(t, u)$ - the complex matrixes reversed to the cross correlation matrixes of the receive vector $\bar{U}(t)$ for the hypothesis of the only noise observation $R(t, u)$ and $k$-signal against $R_{k}(t, u)$ noise background.

Due to the noise and the diffuse component of the receive signal are $\bar{U}_{k 0}(t)$ independent random processes,

$$
R_{k}(t, u)=R_{k 0}(t, u)+R_{0}(t, u)
$$


where

$$
R_{k 0}(t, u)=\left\langle 0,5 \bar{U}_{k \sigma}(t)+\bar{U}_{k \sigma}^{*}(u)\right\rangle
$$

- the correlation function matrix of diffuse components of the receiver signal vector.

For the determining the matrix form $Q_{0}(t, u)$ and $Q_{k}(t, u)$ is used the integral-matrix inversion equations [5]:

$$
\begin{aligned}
& \int R_{0}(t, u) Q_{0}(u, v) d u=I \delta(t-v) ; \\
& \int R_{k}(t, u) Q_{k}(u, v) d u=I \delta(t-v),
\end{aligned}
$$

where $I$ - the singular diagonal matrix.

For the final addend in (1) the equation holds [6]:

$$
\ln \left(K_{0} / K_{k}\right)=S p \int_{0}^{1} \frac{d A}{A} \iint R_{0}(t, u) Q_{A k}(t, u) d t d u
$$

where $Q_{A k}(u, t)$ - the solution of the integral-matrix equation:

$$
\iint\left[R_{0}\left(t_{1}, u\right)+A R_{k 0}\left(t_{1}, u\right)\right] Q_{A k}(u, v) R_{0}\left(v, t_{2}\right) d u d v=A R_{k 0}\left(t_{1}, t_{2}\right)
$$

Providing the white noise with the diagonal matrix of spectral density $N_{0}$ equations $(4-7)$ are simplified:

$$
\begin{gathered}
Q_{0}(t, u)=N_{0}^{-1} \delta(t, u) \\
\int R_{k 0}(t, u) Q_{k}(u, v) d u+N_{0} Q_{k}(t, v)=I \delta(t-v) \\
\ln \left(K_{0} / K_{k}\right)=S p \int \frac{d A}{A} \int N_{0} Q_{A k}(t, t) d t \\
N_{0} Q_{A k}(t, v) N_{0}+A \int R_{k 0}(t, u) Q_{A k}(u, v) N_{0} d u=A R_{k 0}(t, v) .
\end{gathered}
$$

Using the references [4], we search for matrix $Q_{k}(t, u)$ in the form

$$
Q_{k}(t, u)=N_{0}^{-1}\left[I \delta(t-u)-Q_{k 0}(t, u)\right]
$$

In this case the equation (9) is transformed as follows:

$$
\int R_{k 0}(t, u) N_{0}^{-1} Q_{k 0}(u, v) d u+Q_{k 0}(t, v)=R_{k 0}(t, v) N_{0}^{-1} .
$$


The comparison of (11) with (13) shows that the matrix $Q_{k 0}(t, u)$ may be found by the equation (11) with $A=1$,

$$
Q_{k 0}(t, u)=N_{0} Q_{A k}(t, u)_{\mid A=1} .
$$

Substitute (8) in (1) receive:

$$
\begin{aligned}
I_{k}[\bar{U}(t)]=0,5\left\{\int \int \left[\bar{U}^{*}(t)\right.\right. & \left.-\bar{U}_{k \sigma}^{*}(t)\right] N_{0}^{-1} Q_{k 0}(t, u)[\bar{U}(u)- \\
& \left.-\bar{U}_{k \sigma}(u)\right] d t d u-\int \bar{U}_{k \sigma}^{*}(t) N_{0}^{-1} \bar{U}_{k \sigma}(t) d t+ \\
& \left.+2 \operatorname{Re}\left[\int \bar{U}_{k \sigma}^{*}(t) N_{0}^{-1} \bar{U}(t) d t\right]-\ln \left(K_{0} / K_{k}\right)\right\}
\end{aligned}
$$

The final equation presents one of possible former structure variants of forming the likelihood ratio algorithm for $k$-hypothesis (Fig. 1). The base of the former $I_{k}[\bar{U}(t)]$ consists of two correlation channels according to (15) and Fig.1. The correlation of the received implementation of the vector $\bar{U}(t)$, which is normalized to the noises power, with the vector of relevant signal, generated by separate determinate components of $k$-class signal is calculated within the first channel $\bar{U}_{k \sigma}(t)$. The second channel has the $\bar{U}(t)-\bar{U}_{k \sigma}(t)$ correlation of the difference signal with the vector

$$
\int Q_{k 0}(t, u)\left[\bar{U}(u)-\bar{U}_{k \sigma}(u)\right] d u
$$

which is the estimation of the diffusive component of the receive signal in the assumption of $k$-class signal observation.

Essentially the paper proposes estimation-correlation algorithm of the likelihood ratio forming, i.e. the sufficient statistics vector for the decision-making of the observed signals classes.

The given algorithm provides optimal reception and processing of fluctuating signal in the case of distortions and interferences.

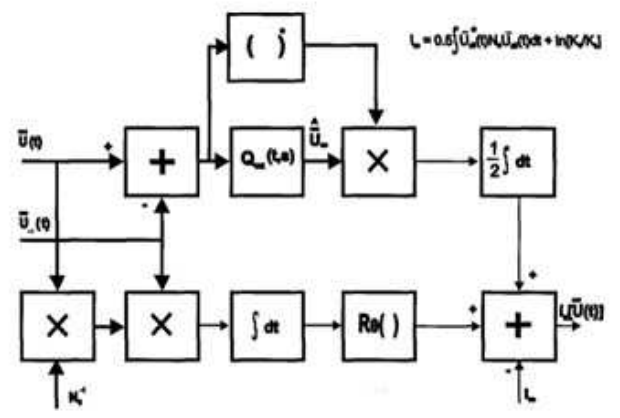


Figure 1: Schematic structure of forming the likelihood ratio logarithm

The majority of potential opportunities of detection task solution are characterized by the probabilities of correct $\left(P_{c o r}\right)$ and wrong $\left(P_{\text {err }}\right)$ decisions. The accurate analytical estimation of these probabilities may be fulfilled only in simple individual cases. In these cases the usage of Kailath [7] bound ratios is more appropriate for the obtaining of quantitative estimations of probable system work characteristics. These ratios have been added for the check of two hypothesis and in this paper are spread for their arbitrary number.

During the check of $(M+1)$ hypothesis the probability of wrong solution equals:

$$
P_{\text {err }}=\sum_{i=1}^{M+1} p_{i} \sum_{\substack{j=1 \\ j \neq 1}} p(j / i)
$$

where $p_{i}$ - a prior probability of $i$-hypothesis, $p(j / i)$ - the probability of finding for $j$-hypothesis in the case when 1 hypothesis is true.

This formula is presented in the next form:

$$
P_{\text {err }}=\sum_{i=1}^{M} \sum_{j=i+1}^{M+1}\left[p_{i} p(j / i)+p_{j} p(i / j)\right]
$$

The next step we suppose the solution of finding for any class is taken with the usage of the minimum criterion of full error probability.

The high error bound er (17) is found using the method [5] with the check of many hypotheses:

$$
P_{e r r} \leq \sum_{i=1}^{M} \sum_{j=i+1}^{M+1}\left[p_{i} C h_{i j}^{F}+p_{j} C h_{i j}^{M}\right]
$$

The latter hypothesis $C h_{i j}^{F}$ and $C h_{i j}^{M}$ - Chernoff bounds for the probability of false alarm and target skip during the check of the pair $i$ and $j$ hypotheses independently on other hypotheses.

There should be noted the error estimation given by the inequation (18) may be much overstated but the accuracy estimation decreases with the increase of the number of competing hypotheses in a general case.

The finding of the lower bound error probability of the correct classification (16) is advisable in these conditions. This bound for the binary detection is coined by Kailath [7]. This paper generalizes the discussed bound in the case of the arbitrary hypotheses number. 
The equation (16) is used in the search of the lower bound of error probability. The sum of $j$ in the right of the part of the equation is the probability of wrong solution with observation of $i$-class target. This error appears when just one of hypotheses has the following inequation:

$$
l_{i j}>l_{i i}(i \neq j)
$$

where

$$
l_{i j}=\ln \left(\frac{W_{j}(U)}{W_{i}(U)}\right)
$$

- the likelihood ratio logarithm during the check of $j$ and $i$ hypotheses; $W_{j}(U)$ and $W_{i}(U)$ - the probability density of observed data vector values $U$ on $j$ and $i$ hypotheses. Suppose $A_{j}$ event equals the fulfillment of the condition (19). The error probability in the observation of $i$-class target is the sum probability of events:

$$
\sum_{\substack{j=1 \\ j \neq 1}}^{M+1} P(j / i)=P\left[\sum_{\substack{j=1 \\ j \neq 1}}^{M+1} A_{j}\right] .
$$

Using the formula for the evaluation of the sum probability of dependent events we prove the true inequation:

$$
P\left[\sum_{\substack{j=1 \\ j \neq 1}}^{M+1} A_{j}\right] \geq \max _{\substack{j \neq i \\ j \neq i}} P\left[A_{j}\right] .
$$

In this equation $p\left[A_{j}\right]$ is the probability of finding for $i$-class targets providing the $i$-class target is true during the check of only this alternative pair. Label this inequation by $P_{2}(j / i)$ and using (22) write the inequation for the error probability (16):

$$
P_{\text {err }} \geq \sum_{i=1}^{M+1} P_{i} P_{2}(j / i),
$$

where $j$ value has the only restriction:

$$
j \neq i \text {. }
$$

The inequation (23) allows estimation of the lower frontier of error probability during the check of more than two hypotheses. The attention is given to the 
following task (generalization of the arbitrary classes numbers is analogous) due to the interest in the recognition of three classes.

Using (22) and (23), write the evident inequations:

$$
\begin{aligned}
& P_{\text {err }} \geq P_{1} P(2 / 1)+P_{2} P_{2}(3 / 2)+P_{3} P_{2}(1 / 3), \\
& P_{\text {err }} \geq P_{1} P(3 / 1)+P_{2} P_{2}(1 / 2)+P_{3} P_{2}(2 / 3) .
\end{aligned}
$$

Adding the right and the left parts of these inequations, we get:

$$
P_{\text {err }} \geq 0.5 \sum_{i=1}^{2} \sum_{j=i+1}^{3}\left[P_{i} P_{2}(j / i)+P_{j} P_{2}(i / j)\right] .
$$

The equation within square brackets (26) is the error probability during the solution of $i$ and $j$ hypotheses check. For the error estimation use the method [5], [8]. It is important to take into account that the hypothesis formulation with numbers $i$ and $j$ does not form the complete group of events, i. e.

$$
P_{i}+P_{j} \neq 1 \text {. }
$$

Using the minimum error criterion, the searching probability is presented graphically in the sum of two hatched areas $C$ and $D$ Fig. 2 (the figure shows posterior densities of probabilities for two observed hypotheses). According to the figure there are written two equations:

$$
\begin{aligned}
& \int P_{i} W_{i}(U) d U=P_{i}=A+C+D, \\
& \int P_{j} W_{j}(U) d U=P_{j}=B+C+D .
\end{aligned}
$$

Adding them, we receive the next:

$$
A+B+2(C+D)=P_{i}+P_{j}
$$

It is easily noted, that:

$$
A+B=\int\left|P_{i} W_{i}(U)-P_{j} W_{j}(U)\right| d U
$$

Consequently, the sought probability of error equals:

$$
C+D=0.5\left\{P_{i}+P_{j}-\int\left|P_{i} W_{i}(U)-P_{j} W_{j}(U)\right| d U\right\} .
$$


For the integral estimation (31) use Schwarz inequality:

$$
\begin{aligned}
& {\left[\int\left|P_{i} W_{i}(U)-P_{j} W_{j}(U)\right| d U\right]^{2} \leq\left\{\int \mid\left[P_{i} W_{i}(U)\right]^{\frac{1}{2}}-\right.} \\
& \left.\quad-\left.\left[P_{j} W_{j}(U)\right]^{\frac{1}{2}}\right|^{2} d U\right\} *\left\{\int\left|\left[P_{i} W_{i}(U)\right]^{1 / 2}+\left[P_{j} W_{j}(U)\right]^{1 / 2}\right|^{2} d U\right\} .
\end{aligned}
$$

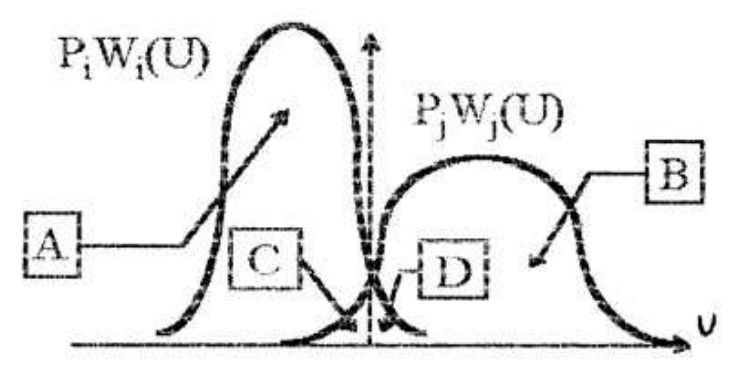

Figure 2: Revisiting the lower bound of the error probability

After the reformation of the right part (32) we get:

$$
(A+B)^{2} \leq\left(P_{i}+P_{j}\right)^{2}-4\left\{\left(P_{i} P_{j}\right)^{1 / 2} \int\left[W_{i}(U) W_{j}(U)\right]^{1 / 2} d U\right\}^{2}
$$

Consequently

$$
P_{i} P_{2}(j / i)+P_{j} P_{2}(i / j)=C+D \geq K l_{i j},
$$

where

$$
\begin{aligned}
K l_{i j}=0.5\left\{P_{i}\right. & +P_{j}- \\
& \left.-\sqrt{\left(P_{i}+P_{j}\right)^{2}-4\left[\left(P_{i} P_{j}\right)^{1 / 2} \int\left[W_{i}(U) W_{j}(U)\right]^{1 / 2} d U\right]^{2}}\right\},
\end{aligned}
$$

This formula is presented in the next form:

$$
K l_{i j}=0.5\left\{P_{i}+P_{j}-\sqrt{\left(P_{i}+P_{j}\right)^{2}-4\left\{\left(P_{i} P_{j}\right)^{1 / 2} \exp \left[\mu_{i j}(0.5)\right]\right\}^{2}}\right\}
$$

where

$$
\mu_{i j}(s)=\ln \left(\int_{\infty}^{\infty}\left[W_{j}(U)\right]^{S}\left[W_{i}(U)\right]^{1-S} d U\right)
$$

The equation (35) demonstrates the synthesis of Kailath bounds for $i$ and $j$ hypotheses that does not form the exhaustive events. 
Finally write (26) in the next form

$$
P_{\text {err }} \geq 0.5 \sum_{i=1}^{2} \sum_{j=i+1}^{3} K l_{i j} .
$$

By analogy the lower bound of error probability for any number of competing hypotheses is found using (26) and (27). In the general case of $M+1$ hypotheses check we have the next:

$$
P_{\text {err }} \geq \frac{1}{M} \sum_{i=1}^{M} \sum_{j=i+1}^{M+1} K l_{i j} .
$$

The above calculations are received for the cases when observed data is the scalar quantity. However, all results are true if the observed data has vector character. Only the equation (37) changes for the logarithm of the moment generatrix as follows:

$$
\mu_{i j}(s)=\ln \left(\int_{\infty}^{\infty}\left[W_{j}(U)\right]^{S}\left[W_{i}(U)\right]^{1-S} d U\right) .
$$

\section{Conclusion}

The paper presents the optimal (within the given conditions) algorithm of processing the observable data with the target of signal presence, its parameters and the class of received signal. The specialization of received algorithm for the certain practical task allows obtaining of the structure chart of the processor which implements the practical algorithm of solving the detection task. Furthermore, the paper shows the strategy of probabilistic characteristics of the classification accuracy.

\section{References}

[1] L.G. Dorosinskiy, The research of the distributed objects' radar image recognition algorithms, Applied and Fundamental Studies, Proceedings of the $2^{\text {st }}$ International Academic Conference, Vol. 1, St Louis, Missouri USA (2013).

[2] L.G. Dorosinskiy, Invariants for the radar image classification. Applied and Fundamental Studies. Proceedings of the $2^{\text {st }}$ International Academic Conference. Vol. 1, St Louis, Missouri USA (2013). 
[3] L.G. Dorosinskiy, Radar Signals Class Recognition Algorithm Synthesis Applied and Fundamental Studies, Proceedings of the $4^{\text {st }}$ International Academic Conference, Vol. 1, St Louis, Missouri USA (2013).

[4] L.G. Dorosinskiy, Synthesis of Signal Flow Group Parameter Optimal Indicator, Proceedings of the $4^{\text {st }}$ International Academic Conference, Vol. 1, St Louis, Missouri USA (2013).

[5] G. Van Tris, Theory of detection, assessments and modulation, Vol. 1, Soviet radio, Moscow (1972).

[6] Y. D. Shirman, V. N. Manzhos, Theory and technology of radar data processing on the interference background, Radio nad Telecommunication, Moscow (1981).

[7] T. Kailath, The divergence and bhattacharya distance measures in signal selection, IEEE Trans., COM-15 (1967), 52-60.

[8] K. Fukunaga, Introduction to statistical pattern recognition, Science, Moscow (1979). 